\title{
邻甲亚基环己二烯酮在天然产物合成中的应用
}

\author{
艾文英 ${ }^{a}$ 廖道红 ${ }^{a}$ 雷晓光 ${ }^{*, b}$ \\ $\left({ }^{a}\right.$ 天津大学药物科学与技术学院 天津 300072) \\ ${ }^{b}$ 北京大学化学与分子工程学院 化学生物学系 北京 100871)
}

\begin{abstract}
摘要 邻甲亚基环已二烯酮, 也被统称为 ortho-Quinone methides (o-QMs), 是由 Fries 课题组在 1907 年提出的, 是一个 半衰期极短、反应活性极高的有机活泼中间体. 它倾向于快速地再芳构化, 这也是它极不稳定的主要原因. 其再芳构化 过程主要通过三个途径: 第一, 和亲核试剂发生 Michael Addition; 第二, 和 $2 \pi$ 体系底物发生 Hetero-Diels-Alder reaction; 第三, 经过氧杂- $6 \pi$-电环化得到苯并吡喃结构. 在天然产物有机合成中, 邻甲亚基环已二烯酮中间体时常经过第 二和第三这两种再芳构化的过程来构建核心骨架, 具有高效便捷的反应特性. 作为有机活泼中间体的新成员, 邻甲亚 基环已二烯酮与卡宾、自由基、碳正离子等活性中间体类似，在有机合成中都具有重要的作用. 在这里，综述了2009 2014 年间邻甲亚基环已二烯酮中间体在天然产物有机全合成中的重要应用.
\end{abstract}

关键词 邻甲亚基环已二烯酮; 天然产物; 有机合成

\section{Applications of ortho-Quinone Methides in the Synthesis of Natural Products}

\author{
Ai, Wenying ${ }^{a} \quad$ Liao, Daohong $^{a} \quad$ Lei, Xiaoguang ${ }^{*, b}$ \\ ( ${ }^{a}$ School of Pharmaceutical Science and Technology, Tianjin University, Tianjin 300072) \\ $\left({ }^{b}\right.$ Department of Chemical Biology, College of Chemistry and Molecular Engineering, Peking University, Beijing 100871)
}

\begin{abstract}
Methylene cyclohexadienones, which are collectivelly referred to as ortho-quinone methides (o-QMs), were first suggested by Fries in 1907. $o$-QMs are a kind of short-lived and highly reactive versatile intermediates in organic synthesis. The unstability nature of $o$-QMs is due to their propensity to undergo rapid rearomatisation either by Michael addition of nucleophiles or, often more usefully, by cycloaddition with $2 \pi$ partners or via $o x a-6 \pi$-electrocyclisation to give benzopyrans. ortho-Quinone methides were always used in the synthesis of natural products through Michael addition and cycloaddition. As a new member of the family of inherently reactive species, ortho-quinone methides are similar with radicals, carbocations, carbenes, etc. And now using $o$-QMs to form skeletons of natural products has become a very useful stratergy. This review contains the major application in the synthesis of natural products during 2009 to 2014.
\end{abstract}

Keywords ortho-quinone methide; natural products; organic synthesis

\section{1 背景介绍}

邻甲亚基环己二烯酮, 系统命名为 6-甲亚基-环己2,4-二烯酮(6-methylene-cyclohexa-2,4-dienone), 该结构 也被统称为 ortho-Quinone methides ( $o$-QMs), 其结构如 1 (Scheme 1). 1907 年 Fries 课题组 ${ }^{[1]}$ 首次提出了 $o-\mathrm{QMs}$ 这个概念, 并基于这个概念解释了反应过程中产生二聚 和三聚产物的原因. 虽然邻甲亚基环已二烯酮中间体这 个概念很早就被提出, 但是因为它自身的不稳定性、高
反应活性和极短的半衰期，直到几十年后才由 McIntosh 和 Chapman ${ }^{[2]}$ 用低温红外光谱证明了这个活泼中间体的 存在. 而它存在的第一个直接证据则是在 1998 年由 Amouri 等得到的 $o-\mathrm{QM}$ 和 $\mathrm{IrCp}^{*}\left(\mathrm{Cp}^{*}=\right.$ 五甲基环戊二 烯)所形成的 $\pi$-配合物 X-ray 单晶结构 ${ }^{[3]}$. 邻甲亚基环己 二烯酮中间体十分倾向于快速的再芳构化，这决定了它 高活泼性的特点. $o-\mathrm{QMs}$ 主要经过三个途径再芳构化: 第一，和亲核试剂发生 Michael Addition 反应; 第二，和

\footnotetext{
*E-mail:xglei@pku.edu.cn

Received April 21, 2015; revised May 8, 2015; published online May 15, 2015.

Project supported by the National Natural Science Foundation of China (Nos. 21472010, 91313303, 21222209).

国家自然科学基金(Nos. 21472010, 91313303, 21222209)资助项目.
} 
$2 \pi$ 体系底物发生 Hetero-Diels-Alder reaction; 第三, 自 身经过氧杂- $6 \pi$-电环化得到苯并吡喃结构(Scheme 1$)^{[4]}$. 也同样是因为邻甲亚基环己二烯酮极容易再芳构化，在 天然产物的合成中, 尤其是含有苯并螺环、苯并吡喃等 结构的天然产物合成中, 利用 $o-\mathrm{QMs}$ 来构建天然产物 骨架成为了化学合成家的常用策略.

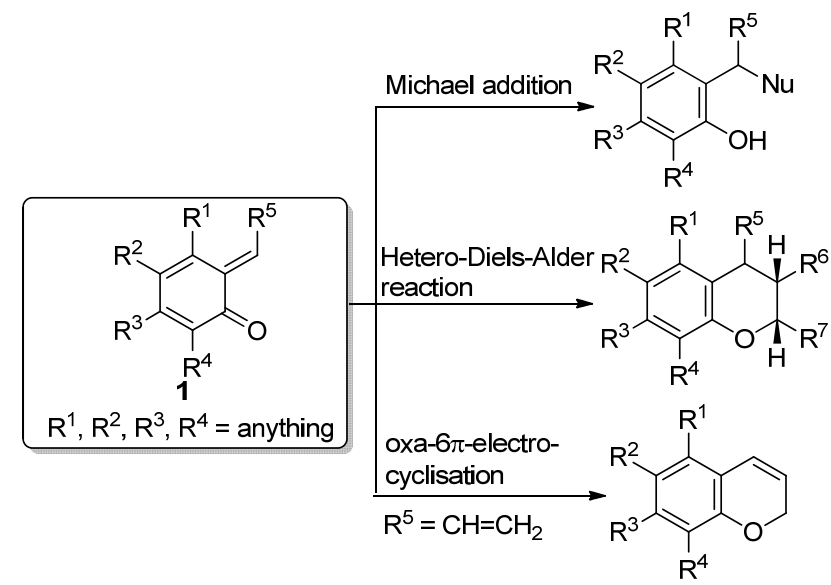

图式 1 邻甲亚基环已二烯酮中间体再芳构化过程

Scheme 1 The rearomatisation of $o$-QMs

\section{1 得到邻甲亚基环己二烯酮中间体的不同前体及方} 法

邻甲亚基环己二烯酮作为一个新型的有机活泼中 间体，和自由基、卡宾、碳正离子等活性中间体类似，其 制备方法有很多. 如 Scheme 2 所示, $o-\mathrm{QMs}$ 可以由不同 的稳定前体通过不同的条件得到, 常见的有四种生成途 径: 第一种, 在加热条件下得到 ${ }^{[5]}$, 例如图中黑色箭头 所对应的前体苯并环醚和邻羟基茮基乙酸酯在加热过
程中很容易经过开环或者 Retro-Michael addition 过程原 位生成 $o-\mathrm{QMs}$; 第二种, 由光照的条件得到 ${ }^{[6]}$, 例如图 中箭头 $\mathrm{a}, \mathrm{b}, \mathrm{c}$ 所对应的前体，很容易在光照条件下经过 自由基途径得到 $o-\mathrm{QMs}$, 其中苯并环醚前体在加热和光 照条件下都可以快速生成邻甲亚基环己二烯酮中间体; 第三种，通过在反应体系中加入亲核试剂 ${ }^{[7]}$ 或者碱 ${ }^{[8]}$ 来 得到, 如图中 d, e, f, g 箭头所对应的带有易离去基团的 $o-\mathrm{QMs}$ 前体，在氟离子或者碱的作用下经过 Retro-Michael Addition 途径可以快速生成目标中间体; 第四种, 通过 Wittig Methylation ${ }^{[9]}$ 、苄基氧化 ${ }^{[10]}$ 或者 Aldol 缩合/脱水化 ${ }^{[11]}$ 等条件得到，如图中箭头 $h, i, j$ 所示 过程. 因此人们可以由不同的 $o-\mathrm{QMs}$ 前体在上述不同 条件下快速构建多种多样的邻甲亚基环已二烯酮中间 体，继而在同一反应体系中，再和其他反应底物一锅高 效便捷地得到不同目标结构骨架.

\section{2 邻甲亚基环己二烯酮中间体可以构建多种多样的 天然产物骨架}

在天然产物的合成中，邻甲亚基环已二烯酮中间体 经常和不同结构的底物经过环加成反应或者 $6 \pi$-电环化 来构建目标分子骨架, Scheme 3 是常见的几种由 $o-\mathrm{QMs}$ 出发构建的结构骨架类型 ${ }^{[12]}$. 例如过程 1 所表示的, 由 2,4-二烯酮-6-亚环己烷自身经过 $6 \pi$-电环化所得到的 $2 \mathrm{H}$-苯并吡喃结构; 还有过程 2、过程 3 所表示的由邻甲 亚基环已二烯酮作为双烯体，由五元或者六元环外烯烃 作为亲双烯体，经过 Hetero-Diels-Alder reaction 所得到 的苯并螺缩酮结构; 因为环外烯烃自身的不稳定性, 特 别容易异构为环内烯烃, 所以当异构化的环内烯烃作为 亲双烯体时得到的是三个并在一起的环结构(如过程

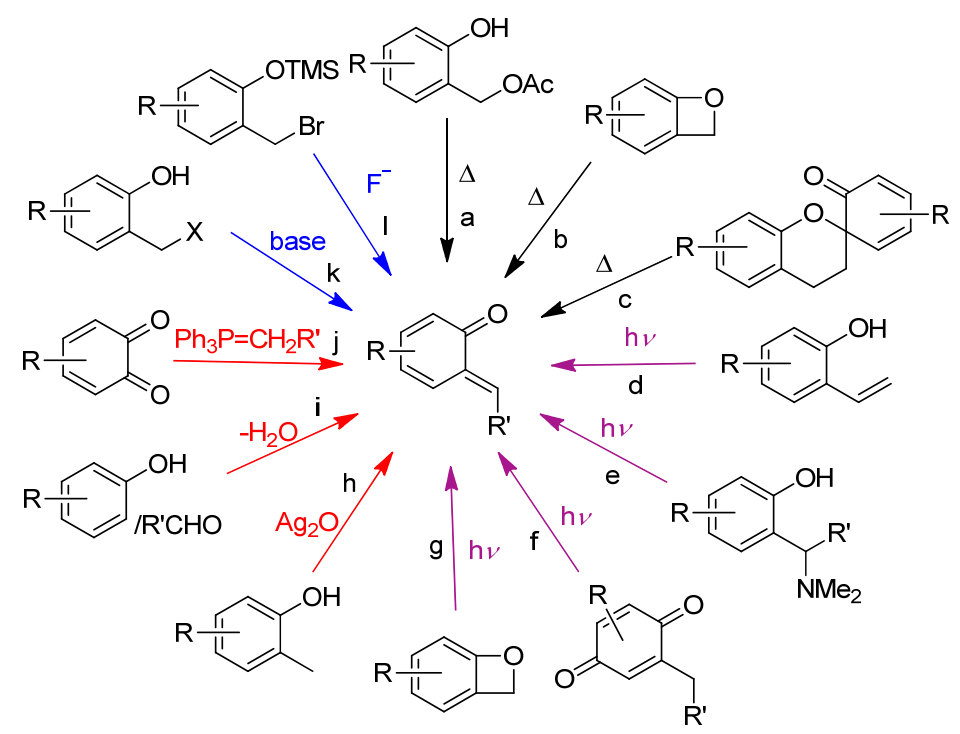

图式 2 构建 $o-\mathrm{QM}$ 的方法

Scheme 2 Methods of forming an $o-\mathrm{QM}$ 


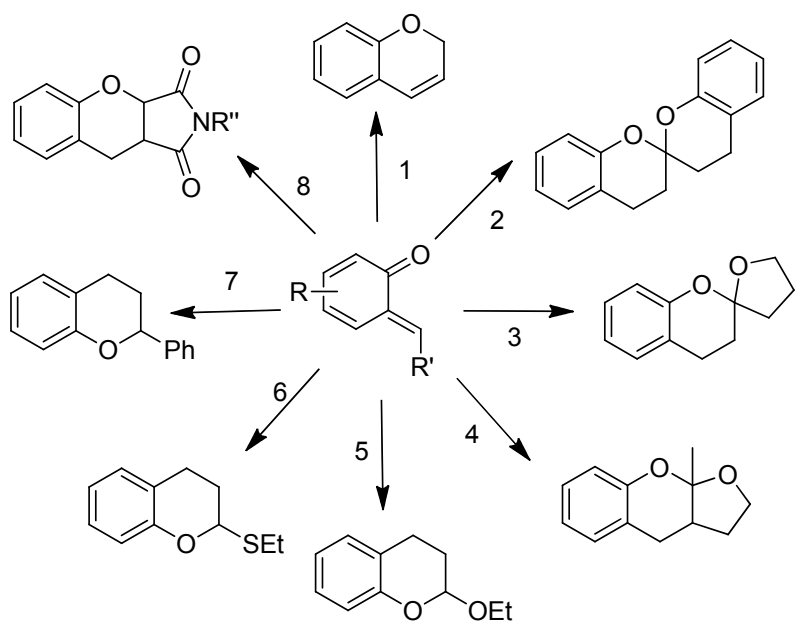

图式 3 邻甲亚基环已二烯酮中间体构建的不同骨架

Scheme 3 The skeleton formed by $o-\mathrm{QMs}$

4 所示); 还因为邻甲亚基环己二烯酮中间体的缺电子 性, 使得它和高富电子烯烃的反应通常是十分高效成功 的, 例如过程 5、过程 6 以及过程 7 所表示的, 由乙烯基 乙基醚、乙烯基乙基硫醚或者苯乙烯作为亲双烯体时, 通常会十分迅速高效的经过 Hetero-Diels-Alder reaction 得到苯并吡喃结构; 邻甲亚基环己二烯酮中间体的高活 性还体现在当它和缺电子的烯烃发生反应时同样是可 行的, 例如过程 8 所表示的, 当琥珀酰亚胺作为亲双烯 体时, 也可以经过 Hetero-Diels-Alder reaction 得到苯并 杂环结构. 除了图中所示, 很多杂环化合物也可以和邻 甲亚基环已二烯酮中间体发生反应，得到不同的骨架结 构, 在此不再一一列举. 由此可见, 在天然产物有机合 成中，应用邻甲亚基环已二烯酮中间体来构建目标骨架 结构是一种很好的策略, 这不仅体现在它可以构建的结 构多样化, 还体现在其高效性, 很多复杂结构都可以利 用这一中间体一锅发生多步串联反应，同时构建多个环 结构, 这对于实现绿色化学所提倡的 “原子经济性” 具 有重大意义.

\section{3 邻甲亚基环己二烯酮中间体在天然产物合成中的 首次应用}

1971 年, Chapman 课题组 ${ }^{[13]}$ 在 Carpanone (5) 的仿生 合成中首次将 $o-\mathrm{QMs}$ 应用于有机全合成, 自此, 开创了 $o$-QMs 在有机合成中应用的新时代(Scheme 4). Carpanone 是从 Carpano 树的树皮中分离出来的, 具有五个 不对称手性中心, 分子中没有对称元素, 这对于它的有 机合成造成了极大的挑战. 在这个工作中, Chapman 及 其同事首先由起始原料 2 经过双键异构化得到烯烃 3 , 然后化合物 3 经过氧化偶联反应构建了 $o-\mathrm{QM}$ 中间体 $\mathbf{4}$, 然后在加热条件下, 中间体 4 自身发生环加成反应, 最
终以 46\%的产率得到了天然产物 Carpanone (5). 这种高 效便捷地构造天然产物策略给许多有机合成化学家带 来了启发，于是越来越多的天然产物都采用类似的策略 经过邻甲亚基环已二烯酮中间体来高效便捷地完成了 全合成工作. Danishefsky ${ }^{[14]}$, Baldwin $^{[15]}$, Snider $^{[16]}$ 等有机 化学家，在这一领域也都做出了极大的贡献.
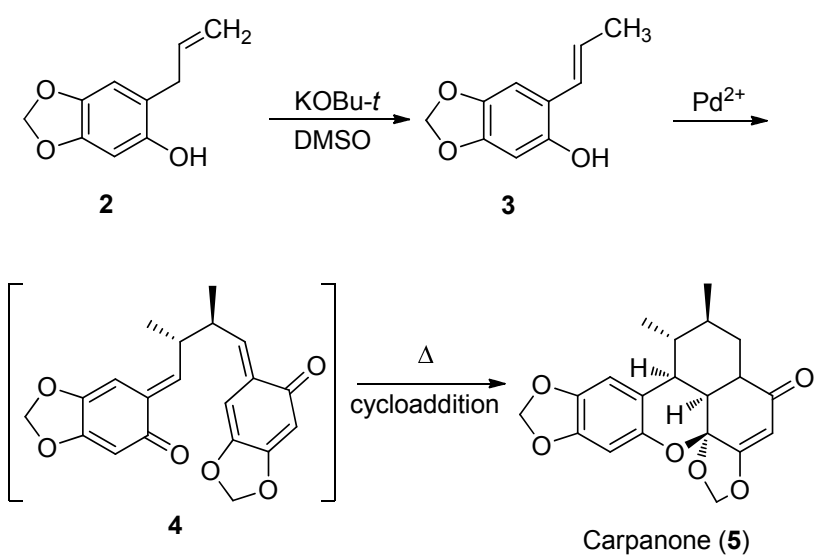

图式 4 天然产物 Carpanone 的合成 Scheme 4 Synthesis of carpanone by chapman

\section{$20-Q M s$ 在天然产物合成中的应用举例}

近几年间，关于 $o-\mathrm{QMs}$ 应用的报道更是层出不穷. 以下，我们将从 2009 年至 2014 年间所报道的实例进行 详细说明.

\section{$2.10-Q M s$ 在 Berkelic acid 全合成中的应用}

2006 年, Stierle 课题组从生活在美国 Berkeley Pit 湖 高酸性、重金属污染恶劣环境中的青霉菌分泌液里分离 到了一种含有四环苯并吡喃螺缩酮骨架的天然产物 Berkelic acid (6b). 研究发现该天然产物对人类卵巢癌 细胞系 OVCAR-3 有很好的生物活性, GI 50 值为 91 $\mathrm{nmol} / \mathrm{L}$, 此外还对两个信号转导酶一一基质金属蛋白酶 MMP-3 和天冬氨酸半胱氨酸蛋白酶 1 也有一定的抑制 活性 ${ }^{[17]}$. 最初, Berkeley Pit 的结构被定为 6a (Scheme $5)^{[18]} .2009$ 年, De Brabander 课题组 ${ }^{[19]}$ 报道了这个天然 产物的全合成. 他们利用邻甲亚基环已二烯酮中间体 7 和 烯醇醚 8 在 银催化剂的作用下, 经过 Hetero-Diels-Alder reaction 构建该天然产物骨架，并将 其结构重新修订为 $6 \mathrm{~b}$ ( Scheme 6), 其中烯醇醚 8 是由炔 基醇 11a 为前体得到的. 这个合成策略的灵感来自于 Berkelic acid 的结构正是天然产物 Spicifernin (9a) 和 Pulvilloric acid (10)的组合, 见 Scheme 5. 从图中我们可 以看出目标分子中红色和蓝色部分分别代表了天然产 物 $9 \mathrm{a}$ 和 10 的类似物. 


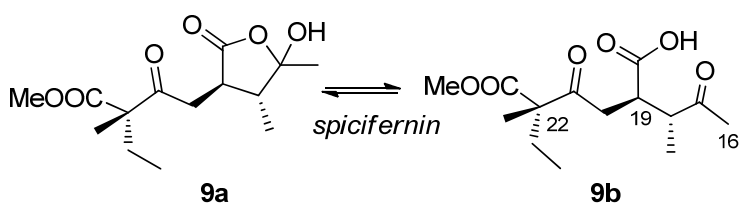

9b

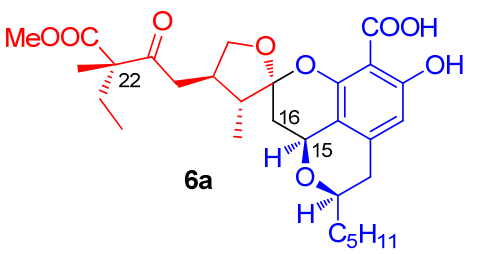<smiles></smiles>
pulvilloric acid (10)

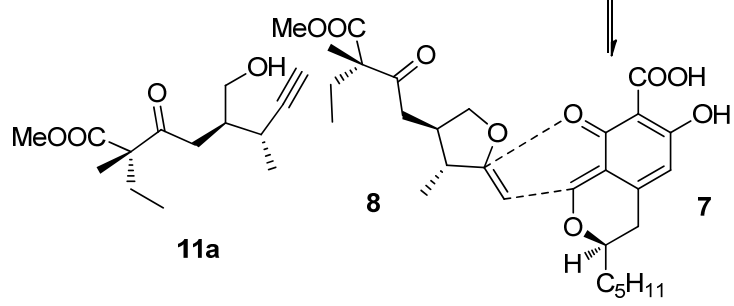

图式 5 De Brabander 提出的 berkelic acid 的合成策略 Scheme 5 The synthetic strategy of berkelic acid suggested by De Brabander

在合成工作中(Scheme 6), 他们首先分别合成了烯 醇醚前体 11a、11b 和邻甲亚基环已二烯酮前体 12. 然 后在催化剂六氟锑酸银的作用下，化合物 11a 和 11b 原 位关环得到五元环外烯醇醚中间体, 同时二酚化合物 12 也在原位发生氧化脱去乙醇反应生成邻甲亚基环己 二烯酮活泼中间体, 之后, 这两个中间体在银催化剂的 作用下一锅发生 Hetero-Diels-Alder reaction 经过过渡态 13 得到 Berkelic acid 甲酯化衍生物 14, 其中邻甲亚基环 己二烯酮是双烯体，烯醇醚是亲双烯体. 然后，以甲苯 为反应溶剂, 在 $\left(\mathrm{Bu}_{3} \mathrm{Sn}\right)_{2} \mathrm{O}$ 的作用下, Berkelic acid 甲酯 化类似物 14 进行选择性脱甲基化，最终以 35\%的产率 得到了目标天然产物 Berkelic acid. 此外, De Brabander 和他的同事们还合成了碳 22 位手性为 $R$ 的 Berkelic acid 的非对映异构体, 通过对比所有的谱图数据, 证实了 Berkelic acid 的绝对构型为 $\mathbf{6 b}$. 在这个工作中, De Brabander 课题组通过设计构建了合适的邻甲亚基环已二 烯酮中间体，一锅发生三步串联反应，高效便捷地构建 了目标天然产物骨架. 这不仅是对这一新型策略的应 用，还提供了一种新型结构的邻甲亚基环已二烯酮中间 体，对于之后的天然产物合成具有重要的指导意义.

\section{$2.2 \mathrm{O}-\mathrm{QMs}$ 在 Guajadial 的仿生合成中的应用}

番石榴在亚洲非洲早已被作为传统的民间药物来 使用，对糖尿病和高血压等疾病都有很好的疗效. 2007 年, 刘吉开课题组 ${ }^{[20]}$ 于番石榴的叶子提取液中分离得

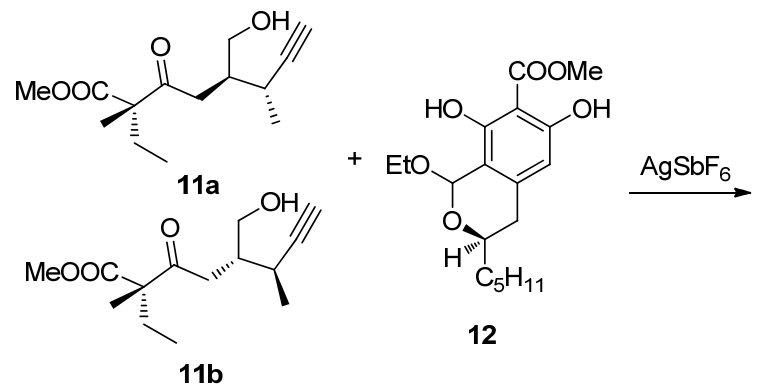

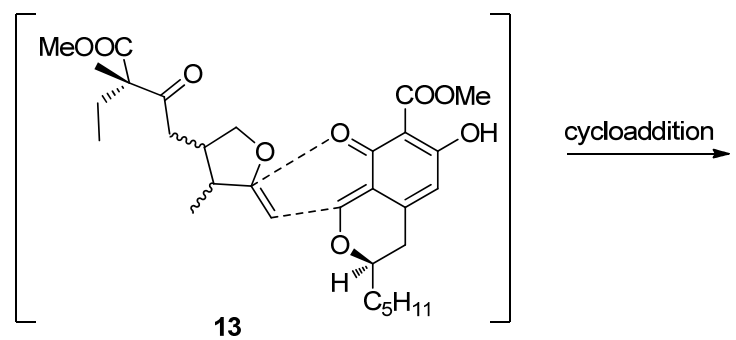

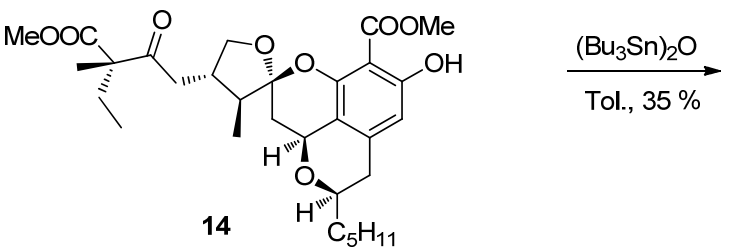

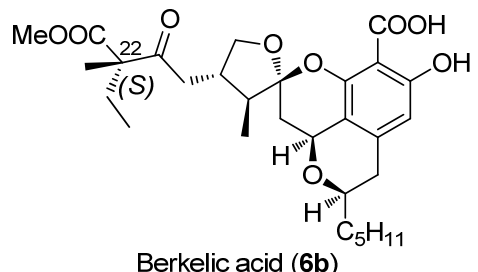

图式 6 De Brabander 合成 berkelic acid 的路线

Scheme 6 Synthesis of berkelic acid by De Brabander

到了番石榴二酫, Guajadial, 即天然产物 20, 它是一种 新的杂萜. 2010 年, Baldwin 和他的同事们 ${ }^{[21]}$ 在 Guajadial 的仿生合成中通过原位生成邻甲亚基环已二烯酮中间 体利用一锅三组分合成策略，快速构建了目标分子骨架 (Scheme 7).

在分离文献中，刘吉开教授及其同事们对番石榴二 醛 20 的生物合成路线进行了推测, 他们认为番石榴二 醛是由石竹烯 19 和 $o$-QM 18 经过 Hetero-Diels-Alder cycloaddtion 得到的(Scheme 7). Baldwin 他们的合成策 略也是基于此得到的灵感. 他们认为生源合成中番石榴 二醛的相对立体化学结构可以由石竹烯 19 的构象部分 控制. 但是，其他的影响因素也要考虑，例如，疏水相 互作用，以及和酶的活性位点之间的 $\pi$-相互作用. 基于 以上分析, Baldwin 及其同事们提出了相应的逆合成分 析，利用苯甲醛 15 和三酚化合物 16 之间的 Knoevenagel 
<smiles>O=Cc1c(O)cc(O)c(C=O)c1O</smiles>

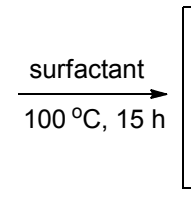<smiles>O=Cc1c(O)c(C=O)c(O)c(C(O)c2ccccc2)c1O</smiles>

17

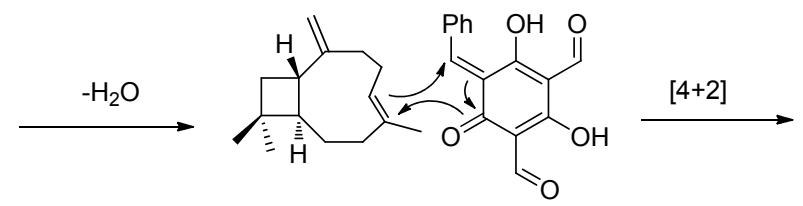
Caryophyllene (19) $\quad 18$

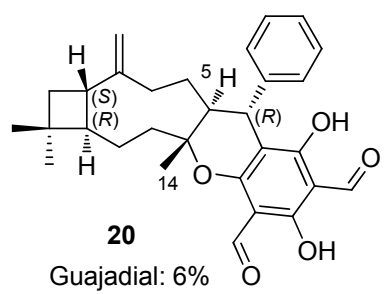

图式 7 Baldwin 课题组对 guajadial 的合成工作

Scheme 7 Synthesis of guajadial by Baldwin and co-workers

condensation 构建邻甲亚基环已二烯酮中间体 18, 继而 在一锅中和 Caryophyllene (19)经过一个三组分串联反 应来完成番石榴二酫的仿生合成. 经过大量的条件篎 选, 他们发现在质量分数为 5\%的 PTS 水溶液表面活性 剂中, 将 16 缓慢滴加到 15 和 19 的混合液里, 在 $100{ }^{\circ} \mathrm{C}$ 条件下反应 $15 \mathrm{~h}$, 可以以 $6 \%$ 的分离产率得到目标天然 产物番石榴二醛 Guajadial (20). 此外, 他们通过将其乙 腈饱和溶液从室温转移到 $-25{ }^{\circ} \mathrm{C}$, 得到了 Guajadial 的 单晶, 进一步确定了其结构. 这个过程中, 一锅串联了 苯甲醛 15 和三酚 16 之间的 Knoevenagel condensation 和邻甲亚基环已二烯酮中间体 18 与石竹烯 19 之间的 Hetero-Diels-Alder reaction 以一种高效便捷的方式得到 了目标分子. 这个三组分策略并不是合成领域的首例, 在这个工作之前, 2006 年 Bharate 和 Singh ${ }^{[22]}$ 报道的天然 产物 Robustadials A 和 B 的仿生合成中就应用了这一策 略, 在此就不再赘述.

\section{$2.30-Q M s$ 在 $( \pm)$ Schefflone 和 Tocopherol trimers 的} 仿生合成中的应用

邻甲亚基环已二烯酮在天然产物合成中的应用还 体现在 $( \pm)$ Schefflone ${ }^{[23]}$ 和 Tocopherol trimers ${ }^{[24]}$ 的仿生 合成中. 这部分工作由雷晓光课题组 ${ }^{[25]}$ 在 2011 年发表 在 Organic Letters 上, 这也是 $( \pm)$-Schefflone 的首次仿生 全合成报道. ( \pm )-Schefflone 和 Tocopherol trimers 是有着 螺-苯并吡喃型三聚骨架的天然产物, 结构复杂. 从生 源上来讲, 这些天然产物可以从邻甲亚基环已二烯酮单 体出发经过 Hetero-Diels-Aler 串联反应得到. 经过前体
篮选雷晓光及其同事发现, $o$-QM 前体 21 在氧化剂氧化 银的作用下, 以苯为溶剂, 室温下反应 $16 \mathrm{~h}$, 可以得到 $o$-QMs 中间体 22, 然后一锅串联发生两次 Hetero-Diels-Aler cycloaddtion 以 $72 \%$ 的产率得到天然产 物 Schefflone (25) (Scheme 8). 此外, 他们还通过用乙烯 基乙基醚作为亲双烯体捕捉到了 $o-\mathrm{QM}$ 中间体 22, 证实 了 $o-\mathrm{QM}$ 中间体 22 的存在.
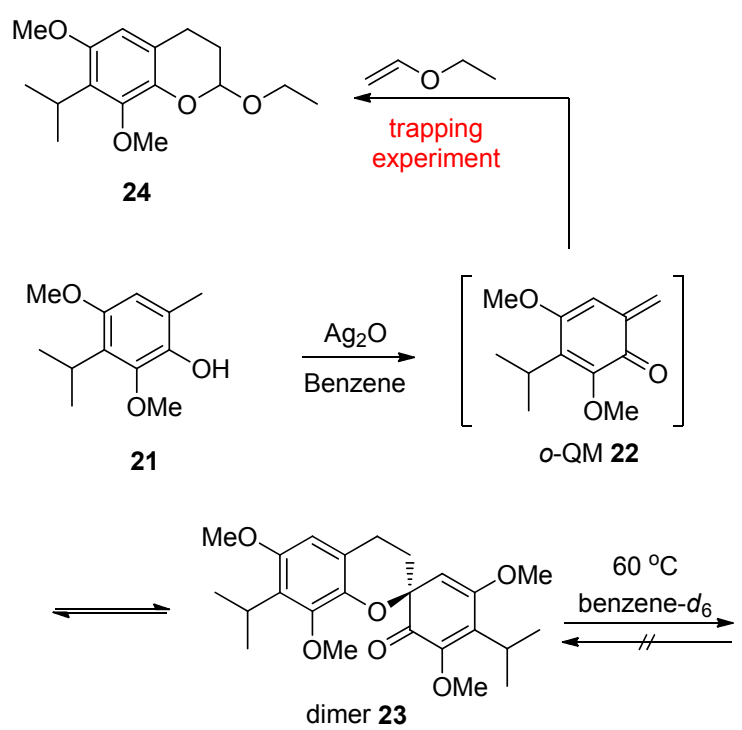<smiles>COC1=C(C(C)C)[C@@]2(OC)Oc3c(cc(OC)c(C(C)C)c3OC)CC[C@H]2[C@@]2(CCc3cc(OC)c(C(C)C)c(OC)c3O2)C1=O</smiles>

( \pm )-Schefflone (25)

图式 8 雷晓光课题组对 schefflone 的合成工作

Scheme 8 Synthesis of schefflone by Xiaoguang Lei and co-workers

为了进一步探索反应过程, 他们做了一系列的反应 机理研究实验. 在 LC-MS 的监测下他们发现反应的前 $15 \mathrm{~min}$ 只有二聚体 $\mathbf{2 3}$ 存在, 随后随着三聚体 $\mathbf{2 5}$ 的构建, 23 和 25 在反应体系中的比例不断发生变化, 直到 $22 \mathrm{~h}$ 后比例不再改变. 并且通过核磁试验发现在 $60{ }^{\circ} \mathrm{C}$ 的反 应温度下, 在気代苯中 $\mathbf{2 3}$ 可以转化为 $\mathbf{2 5}$, 但是 $\mathbf{2 5}$ 在同 样条件下却不能转化为 23. 这些试验结果都证明了中 间体 22 的存在, 以及 23 和 22 之间的可逆性互变, 但是 发生在 23 和 22 之间第二次的 [4+2]-Hetero-Diels-Alder cycloaddtion 是不可逆的, 因此最终三聚体 $\mathbf{2 5}$ 是主要产 物(Scheme 8).

得到 Schefflone (25)之后, 雷晓光课题组进一步利 用这一策略, 以 Tocopherol (28)作为邻甲亚基环已二烯 酮前体, 在氧化银的氧化作用下一锅得到了(一)-To- 
copherol trimer (26)和(一)-Tocopherol trimer (27)两个三 聚体，其产率分别是 36\%和 37\% (Scheme 9).

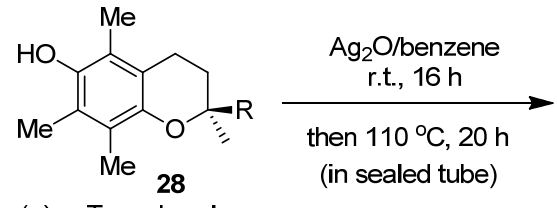

$(+)-\alpha$-Tocopherol

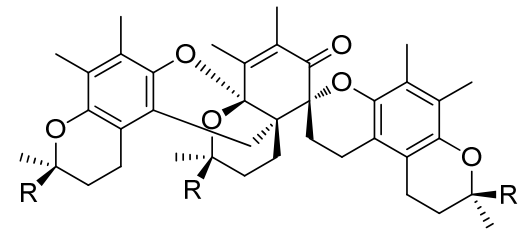

(-)-Tocopherol trimer (26) $36 \%$

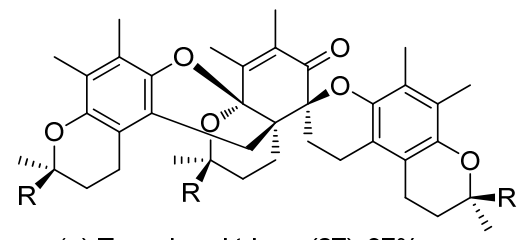

(+)-Tocopherol trimer (27) $37 \%$

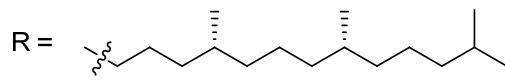

图式 9 雷晓光课题组对 tocopherol trimer 的合成工作 Scheme 9 Synthesis of tocopherol trimer by Xiaoguang Lei and co-workers

\subsection{0 -QMs 在 Tocopherol 合成中的应用}

上一实例中的天然产物 Tocopherol (28)的合成也可 以利用邻甲亚基环已二烯酮作为反应中间体经过 $6 \pi$ 电 环化得到. 2012 年 Wulff 和他的同事 ${ }^{[26]}$ 报道了一种利用 $\alpha, \beta$-不饱和 Fischer卡宾铬复合物和炔丙基醚构建苯并吡 喃结构的新型的有机合成方法, 并利用这种方法设计合 成了 Tocopherol (28) (Scheme 10).

首先, $\alpha, \beta$-不饱和 Fischer 卡宾铬复合物 29 和炔丙基 硅基醚(30)在二氯甲烷中, $60{ }^{\circ} \mathrm{C}$ 条件下, 反应 $24 \mathrm{~h}$, 经 过苯并环化得到中间体 31, 随后通过脱去 $\mathrm{TBSOH}$, 得 到邻甲亚基环已二烯酮中间体 32, 最后, 中间体 32 经 过 $6 \pi$ 电环化以 $85 \%$ 的产率得到了 Tocopherol 前体 33 . 前体 33 经过钯碳氢气还原双键和三氟化硼二甲硫醚复 合物脱甲基两步高产率的得到了目标产物 Tocopherol (28). 从这个工作中我们也可以发现邻甲亚基环已二烯 酮在经过 $6 \pi$-氧杂电环化的过程再芳构化的途径, 更多 地适合含有苯并吡喃结构的天然产物.

\section{$2.50-Q M s$ 在 Hyperguinone $B$ 的合成中的应用}

George 课题组 ${ }^{[27]}$ 在 2010 年同样利用邻甲亚基环已 二烯酮 $6 \pi$ 电环化反应合成了天然产物 Hyperguinone B.
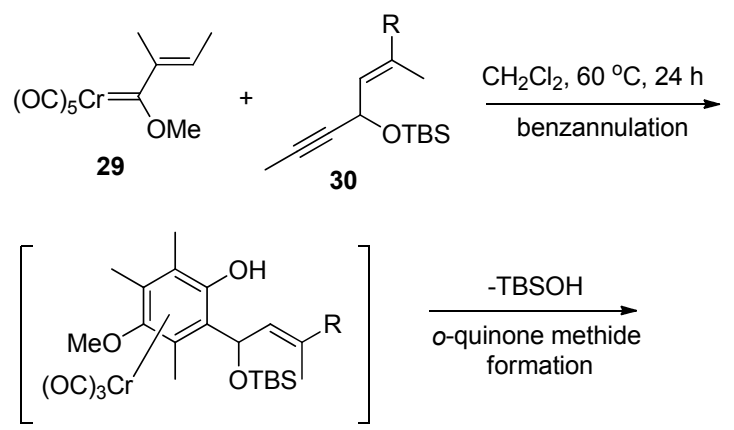

31
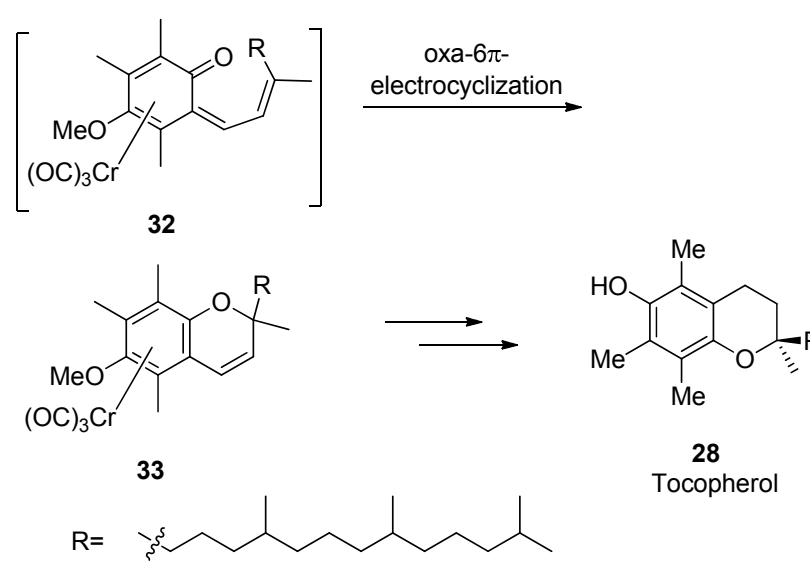

图式 10 Tocopherol 的合成

Scheme 10 Synthesis of tocopherol

Hyperguinone B 是由 Sticher 课题组 ${ }^{[28]}$ 在 2000 年从巴布 亚新几内亚的传统药材巴布亚金丝桃的叶子中分离得 到的, 对很多细菌都表现出来潜在的抗菌活性, 例如: 醋样芽胞杆菌、表皮葡萄球菌和藤黄微球菌. 从结构上 来说, Hyperguinone B 具有双环结构, 其中包含一个 2,2二甲基- $2 H$-吡喃环.

在合成 Hyperguinone B 的过程中(Scheme 11), 首先 George 及其同事以间苯三酚(34)为起始原料分别经过 Friedel-Crafts Acylation ${ }^{[29]}(83 \%)$ 、异戊烯基化 ${ }^{[30]}(39 \%)$ 和 C-甲基化(78\%)合成了关键中间体异戊烯间苯三酚 35a, 苯三酚 35a 是一个很不稳定的中间体, 在溶液中, 是以 35a 和 35b 两个互变异构体按 $3: 1$ 的比例混合存在的. 但是在其正己烷溶液中得到的晶体是化合物 $35 \mathrm{a}$, 经过 X-ray 确定了其化学结构. 然后, 35a 在 TEMPO/ $\mathrm{PhI}(\mathrm{OAc})_{2}$ 作用下经过选择性氧化得到邻甲亚基环已二 烯酮中间体 36, 随后 36 经过分子内 $6 \pi$ 电环化反应最终 以 $73 \%$ 的产率得到了目标天然产物 Hyperguinone B (37). 这是应用邻甲亚基环已二烯酮中间体的 $6 \pi$-氧杂 电环化反应构建苯并吡喃结构的又一实例. 因此, 在以 后的合成工作中，对于含有苯并吡喃结构的天然产物我 们可以考虑利用这一策略来构建分子骨架. 
<smiles>CC(C)=CCC1=C(O)[C@H](CC=C(C)C)C(=O)C(C(=O)C(C)C)=C1O</smiles><smiles>CC(C)=CC[C@]1(C)C(=O)C(C(=O)C(C)C)=C(O)c2cc(C)ccc21</smiles>

图式 11 Hyperguinone B 的合成

Scheme 11 Synthesis of hyperguinone B

\section{$2.60-Q M s$ 在 Heliespirones A 和 $C$ 的合成中的应用}

研究发现分离于向日葵叶子提取液的天然产物 Heliespirones A 和 C 具有胚芽鞘抑制作用, 这表明 Heliespirones $\mathrm{A}$ 和 $\mathrm{C}$ 有被开发为新型化感农药的潜力 ${ }^{[31]}$. 从结构上看, Heliespirones $\mathrm{A}$ 和 $\mathrm{C}$ 具有十分少见的氧杂 螺环结构, 这些特性都大大的吸引着化学合成家们. 根 据生源合成推测, Heliespirones $\mathrm{A}$ 和 $\mathrm{C}$ 是分别由各自相 对应的苯并环醚氧化开环得到对应的醌, 再经过分子内 的 1,4-共轭环加成反应得到的 ${ }^{[32]}$. 于是在此基础上, Pettus 课题组 ${ }^{[33]}$ 提出了以下逆合成分析(Scheme 12), 由 关键中间体 41 构建所需要的苯并环醚结构 39, 苯并螺 环 41 则可以由邻甲亚基环已二烯酮中间体 43 和环外烯 烃 42 经过 Hetero-Diels-Alder reaction 得到.

于是, Pettus 及其同事以 45 为原料(Scheme 13), 经 过 Friedel-Crafts Acylation, 1,4-Michael addition 和 Boc 保护三步合成了邻甲亚基环已二烯酮前体 46, 然后 46 在甲基溴化镁的作用下生成邻甲亚基环己二烯酮中间 体 47, 同时与环外烯烃 48 发生 Hetero-Diels-Alder reaction, 以 $81 \%$ 的产率得到目标关键中间体苯并螺环 49. 49 再经过四步化学转化可以得到想要的苯并环醚 $\mathbf{5 0}$, 随后, 50 的芳香环在氧化银的氧化作用下得到二醌 $\mathbf{5 1}$, 在碳酸铯作碱条件下二醌 $\mathbf{5 1}$ 以 $45 \%$ 的产率转化得到了 目标天然产物 ent-heliespirone A (52). 在这一过程中, Hetero-Diels-Alder reaction 虽然只是作为一个中间步骤<smiles>C=CC1CC(C(C)(C)O)OC2(CC(=O)C(C)=CC2=O)C1</smiles>

Heliespirone A (38a) Heliespirone C (38b)

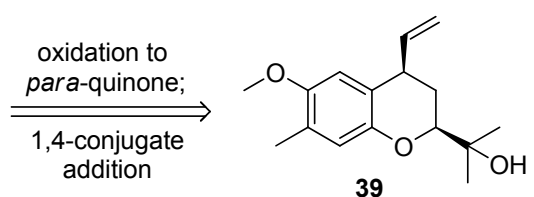

39<smiles>C=CC1CC(C(C)(C)C)Oc2cc(C)c(OCC)cc21</smiles><smiles>[R]C1OC(=O)C(C)(C)O1</smiles><smiles>C=CC=COc1cc(C=O)c(OC=C)cc1C</smiles>

44

图式 12 Pettus 课题组提出的 heliespirones $\mathrm{A}$ 和 $\mathrm{C}$ 的合成策略 Scheme 12 The synthetic strategy of heliespirones A and C suggested by Pettus

构建了中间体苯并螺环结构 $\mathbf{4 9}$, 但是对于很多含有苯 并螺缩酮结构的天然产物, 这一策略不失为一种高效的 合成策略. 例如在前面所提到的 De Brabander 和他的同 事所报道的关于 Berkelic acid 的合成工作, 还有 Pettus 课题组 ${ }^{[34]}$ 所报道的关于 Paecilospirone 的全合成工作都 应用了这一策略高效的构建了天然产物中含有的苯并 螺缩酮结构. 这些工作都值得我们在以后的合成工作中 去借鉴.

\section{$2.70-Q M s$ 在 Variecolortides A 和 B 的全合成中的应 用}

Trauner 课题组在邻甲亚基环己二烯酮中间体的应 用方面也同样做出了重要的贡献. 2011 年, 他们 ${ }^{[35]}$ 报道 了天然产物 Variecolortides A 和 B 的全合成. Variecolortides $\mathrm{A}$ 和 $\mathrm{B}$ 是 2007 年由朱伟明课题组 ${ }^{[36]}$ 从变色曲霉 (Aspergillus variecolor) 的耐盐菌株中分离得到的, 其结 构新颖，拥有罕见的 9,10-葸醌甲基并二氢吡喃稠环结 构, 该四环系统还进一步连接了二酮哌嗪, 产生螺环 $\mathrm{N}, \mathrm{O}-$ 缩醛结构, 此外还有一个吲哚环与二酮哌嗪相连. 在生物活性上, 这类天然产物表现出了适当的细胞毒 性. 


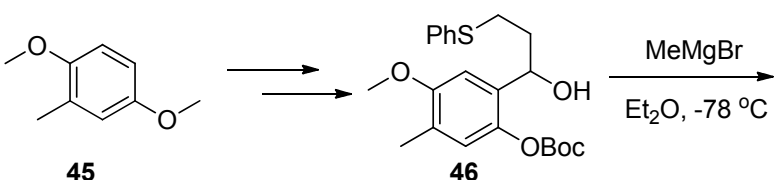

45<smiles>Cc1cc(OC(C)(C)C)c(C([O-])CCP)cc1OCCc1ccccc1</smiles><smiles>CCOc1cc(C(CCS)OCC)c([O-])cc1C</smiles><smiles>CCOC1=CC(=CCCS)C(=O)C=C1C</smiles><smiles>C=C1OCO[C@@H]1C</smiles>

$$
\underset{\text { cycloaddition }}{\left(\stackrel{\overline{\overline{\mathrm{C}}} \mathrm{H}_{2}}{2}\right)_{2} \mathrm{Ph}}
$$

47<smiles>C=CC1C[C@@H](C(C)(C)C=C)[C@H](C(C)(C)O)Oc2cc(C)c(OCC#CC)cc21</smiles>

49<smiles>C=C[C@H](C[C@H](O)C(C)(C)O)C1=CC(=O)C(C)=CC1=O</smiles>

图式 13 Heliespirones A 和 $\mathrm{C}$ 的合成

Scheme 13 Synthesis of heliespirones A and C

通过对天然产物 Variecolortides A 和 B 结构分析可 以发现, Variecolortides A 和 B 是由另外两个天然产物构 成的: Hydroxyviocristin 53(分离于冠突曲霉 Aspergillus cristatus) ${ }^{[37]}$ 和 Isoechinulins $55 \mathbf{a}, 55 \mathbf{b}$ (分离于赤曲霉 Aspergillus ruber $)^{[38]}$. 于是 Trauner 及其同事们提出可以 利用 Hydroxyviocristin (53)原位生成 $o-\mathrm{QM}$ 中间体和 Isoechinulins 55a, 55b 经过串联仿生 Hetero-Diels-Alder reaction 和氧化反应构建目标天然产物的分子骨架螺环 $\mathrm{N}, \mathrm{O}-$ 缩醛结构 ${ }^{[35]}$.

在合成过程 中 (Scheme 14), 天然产物 Hydroxyviocristin (53) 在邻二氯苯中加热会发生 1,5-H迁 移，从而得到邻甲亚基环已二烯酮中间体 $\mathbf{5 4}$, 然后中间 体 54 原位和 55a 或者 55b 发生 Hetero-Diels-Alder reaction 得到苯并吡喃结构产物 56a 和 56b. 随后, 56a 和 56b 在空气作用下发生自动氧化生成双键, 得到目标分 子 Variecolortides A (57a) 和 B (57b), 其产率分别是 48\% 和 $32 \%$. 在这个工作中, Trauner 及其同事以二醌化合物
为前体利用加热的方法得到了邻甲亚基环已二烯酮中 间体，为我们提供了一种新型的邻甲亚基环已二烯酮前 体结构, 和前面提到的氧化、加碱等条件一样是一种重 要的制备邻甲亚基环己二烯酮活泼中间体的条件.<smiles>CC1=CC(=O)c2c(cc3cc(O)cc(O)c3c2O)C1=O</smiles>

Hydroxyviocristin 53<smiles>CC1=CC(O)=C2C(=O)c3c(O)cc(O)cc3C=C2C1=O</smiles>

o-QM (54)

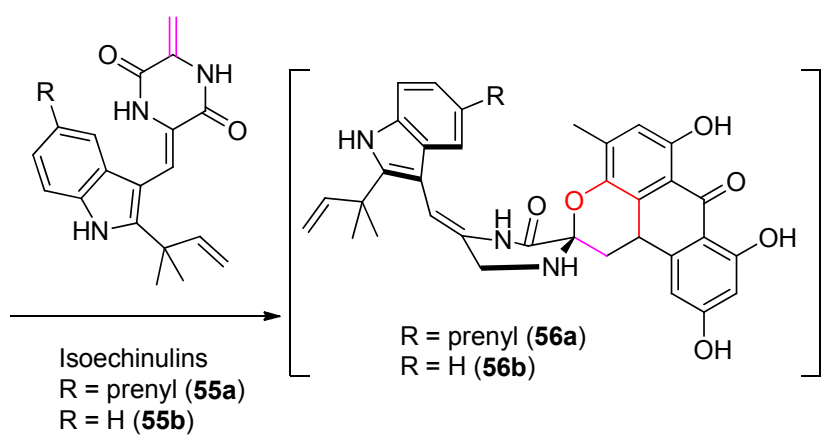<smiles>[R]c1ccc2[nH]c(C(C)(C)C=C)c(C=C3CNC(=O)C4(C=C5C(=C(C)C=C(O)C6=C5c5cc(O)cc(O)c5C6=O)O4)C3)c2c1</smiles>

$\mathrm{R}=$ prenyl; Variecolortide $\mathrm{A}(\mathbf{5 7 a}) 48 \%$ $\mathrm{R}=\mathrm{H} ;$ Variecolortide $\mathrm{B}(\mathbf{5 7 b}) 32 \%$

图式 14 天然产物 Variecolortides A 和 B 的合成 Scheme 14 Synthesis of variecolortides A and B

\section{$2.80-Q M s$ 在 Heliananes 家族天然产物合成工作中的 应用}

2011 年 Pettus 课题组 ${ }^{[39]}$ 报道了天然产物 Heliananes 家族的合成工作, Crews 等 ${ }^{[40]}$ 曾提出这一家族天然产物 的共同生源前体是天然产物 $S$ - $(+)$-Curcuphenol. 于是 $S$ - $(+)$-Curcuphenol 62 的合成工作对整个 Heliananes 家 族天然产物的合成显得至关重要.

在这个工作中，Pettus 和他的同事们利用 Hetero-Diels-Alder reaction 和 Wittig reaction 合成了天然 产物 $S$ - $(+$ )-Curcuphenol (Scheme 15). 首先, 起始原料 2-差基-4-甲基-苯甲醛 58 在过量的甲基溴化镁和二碳酸 二叔丁酯的作用下生成邻甲亚基环己二烯酮中间体 $\mathbf{5 9}$, 该中间体随后被烯基醚 60 所捕获，经过 Hetero-DielsAlder reaction 构建了苯并吡喃结构 61. 61 再经过缩酮水 
解和 Wittig reaction 得到 Heliananes 家族的生源前体天 然产物 $S$ - $(+)$-Curcuphenol 62. 这又是一个构建苯并吡 喃结构的例子, 因此除了经过 $6 \pi$-电环化的方法得到这 一结构外, 利用 Hetero-Diels-Alder reaction 同样是可以 选择的策略之一, 只是要构建的邻甲亚基环己二烯酮前 体有所不同.<smiles>Cc1ccc(C=O)c(O)c1</smiles>

58

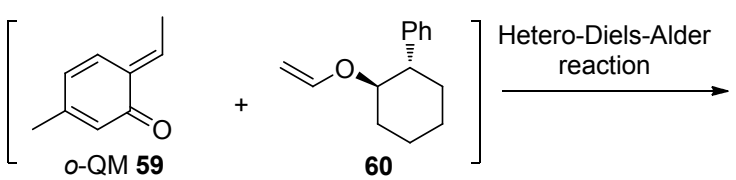<smiles>[R]OC1C[C@H](C)c2ccc(C)cc2O1</smiles>

61 a) $\mathrm{MeMgBr}, \mathrm{Et}_{2} \mathrm{O}$

$60,-40{ }^{\circ} \mathrm{C}$

b) $\mathrm{Boc}_{2} \mathrm{O},-40^{\circ} \mathrm{C}$<smiles>CCCCc1ccc(C(C)CC=C(C)C)c(O)c1</smiles>

62 $S-(+)-C u r c u p h e n o l$

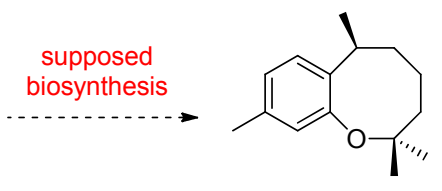

(+)-Helianane 63

图式 15 天然产物 $S-(+)$-Curcuphenol 的合成

Scheme 15 Synthesis of $S$ - $(+)$-curcuphenol

\section{$2.9 \mathrm{O}-\mathrm{QMs}$ 在 Dependensin 的合成工作中的应用}

在众多来自自然界的天然产物中, 含有四氢苯并吡 喃多环母核结构的很多天然产物都具有很好的生物活 性, 有些被用于降血压药, 还有些因其具有良好的细胞 毒性而具有被开发为抗癌药的潜力 ${ }^{[41]}$. 因此如何对这 类天然产物进行全合成也成为了众多化学家关注的热 点. 2011 年 Schaus 课题组 ${ }^{[42]}$ 报道了一种利用铁催化剂催 化 $2 \mathrm{H}$-苯并吡喃发生重排和环加成串联反应的有机合成 方法, 并利用这种合成方法一锅得到了天然产物 Dependensin.

首先经过条件笁选, 他们找到了最优催化剂六水合 三氯化铁, 然后在这一条件下, 起始原料 64 发生开环反 应得到 $Z$ 式邻甲亚基环已二烯酮中间体 65, 65 又迅速的 可逆转化为 $E$ 式邻甲亚基环已二烯酮中间体 66, 这两个 异构体在体系中达到平衡, 随后和体系中存在的 64 发 生 Hetero-Diels-Alder reaction 得到天然产物 Dependensin, 产率是 $62 \%$ (Scheme 16).

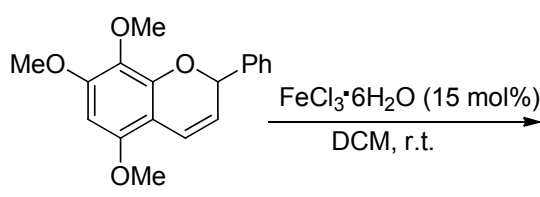

64<smiles>COC1=CC(=O)C(=CC=Cc2ccccc2)C(OC)=C(OC)C1=O</smiles>

66<smiles>COC1=CC(OC)=C(OC)C2O[C@]3(C)c4c(OC)cc(OC)c(OC)c4OC(c4ccccc4)[C@]3(c3ccccc3)[C@H](c3ccccc3)[C@H]12</smiles>

图式 16 天然产物 Dependensin 的合成 Scheme 16 Synthesis of dependensin

在这个工作中所涉及到的邻甲亚基环已二烯酮中 间体 65 是由苯并吡喃 64 开环得到的，而在前面所提到 的 Hyperguinone B 和 Tocopherol 的合成中是由邻甲亚基 环已二烯酮中间体经过 $6 \pi$-电环化来构建苯并吡喃结构, 因此在这一类天然产物中两者可以互为合成前体.

\section{$2.10 O-Q M s$ 在 Spirooliganones $A$ 和 $B$ 的合成工作中} 的应用

流感病毒仍在威胁着人类的生活，例如 2009 年所 爆发的大流感 $\mathrm{H} 1 \mathrm{~N} 1 / 09$ 造成的死亡人数超过了 17700 人. 因此抗病毒药物的发现在人类生活中仍十分必要. 2013 年, 㢎石山等 ${ }^{[43]}$ 从少药八角的根须中提取分离得 到了两个结构新颖的天然产物 Spirooliganones A 和 B $(68 \mathrm{a}$ 和 $68 \mathrm{~b})$. 经过生物活性测定发现这两个天然产物具 有良好的、广泛的抗病毒活性. 而且少药八角在中国民 间被广泛的用于治疗风湿性关节炎. 2014 年，童荣彪课 题组 ${ }^{[44]}$ 完成了 Spirooliganones A 和 B 的不对称全合成. 逆合成分析如下(Scheme 17), 首先采用 Hetero-DielsAlder reaction 由 Diene 71 和 Dienophile 72 来构建螺环 结构前体 70, 这个设计可以一步构建出目标天然产物 Spirooliganones A 和 B 里的 BCDE 四个环. 二醇 69 则由 70 经过 Sharpless asymmetric dihydroxylation 得到. 最后 69 经过苯酚氧化和螺环化过程构建出 A 环, 得到目标 天然产物. 

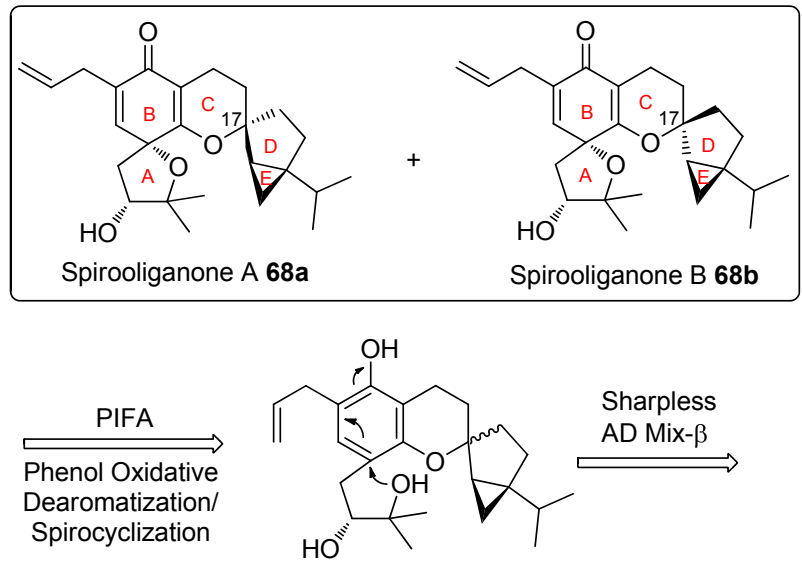

69

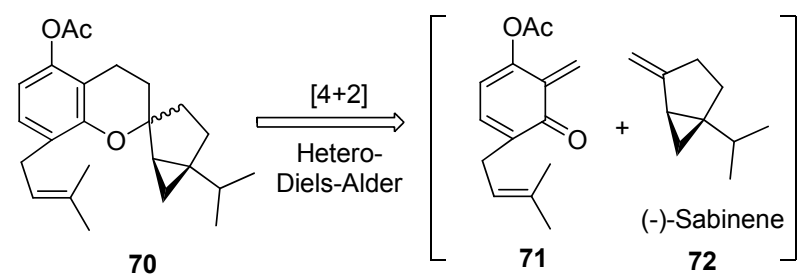

图式 17 童荣彪课题组提出的 Spirooliganones A 和 B 的合成 策略

Scheme 17 The synthetic strategy of spirooliganones A and B suggested by Rongbiao Dong and co-workers.

首先, 他们以 2,6-二羟基苯甲酸 73 为原料经过四步 合成了化合物 74, 之后化合物 $\mathbf{7 4}$ 在以(-)-Sabinene 为 溶剂的体系中回流(约 $\left.160{ }^{\circ} \mathrm{C}\right) 1 \mathrm{~h}$, 通过原位生成邻甲 亚基环已二烯酮中间体和(一)-Sabinene 上的环外双键发 生 Hetero-Diels-Alder reaction, 最终以 79\%的产率得到 了螺环结构 70, 一步构建了 $\mathrm{BCDE}$ 四个环, 其中 70 是 一对非对应异构体的混合物, 其 $d r$ 值是 $1: 1$. 随后, 70 再经过几步结构修饰和转化便可得到目标天然产物 Spirooliganones A 和 B (Scheme 18).

\section{3 总结与展望}

从以上实例中不难看出, 邻甲亚基环已二烯酮这个 活性极高的有机合成中间体在天然产物合成中已经占 据了重要的位置, 并成为了一条十分高效便捷的有机合 成策略, 尤其是在含有螺环、苯并吡喃等结构的天然产 物中, 利用邻甲亚基环已二烯酮来快速的构建骨架结构 更是不失为一种良策.

随着这种策略越来越多的被使用, 邻甲亚基环己二 烯酮前体的种类也越来越多元化，除了文中举例所提到 苯并环醚、2-羟基茮醇、2-羟基甲苯等结构还有很多其 它的前体可以在适当条件下得到邻甲亚基环已二烯酮 中间体. 此外还有很多得到这个活泼中间体的方法也在 不断的得到探索, 归纳起来, 其中常用的条件仍是通过 加热、光照、路易斯酸、氧化等, 具体的条件选择应根<smiles>O=C(O)c1c(O)cccc1O</smiles>

73

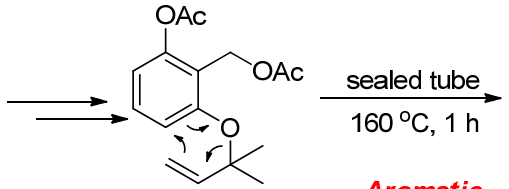

74
Aromatic

Claisen Rearrangement
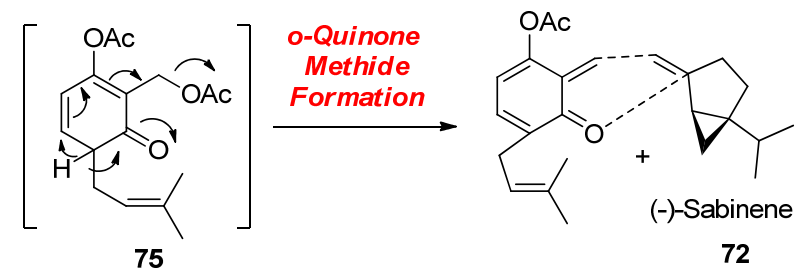

72
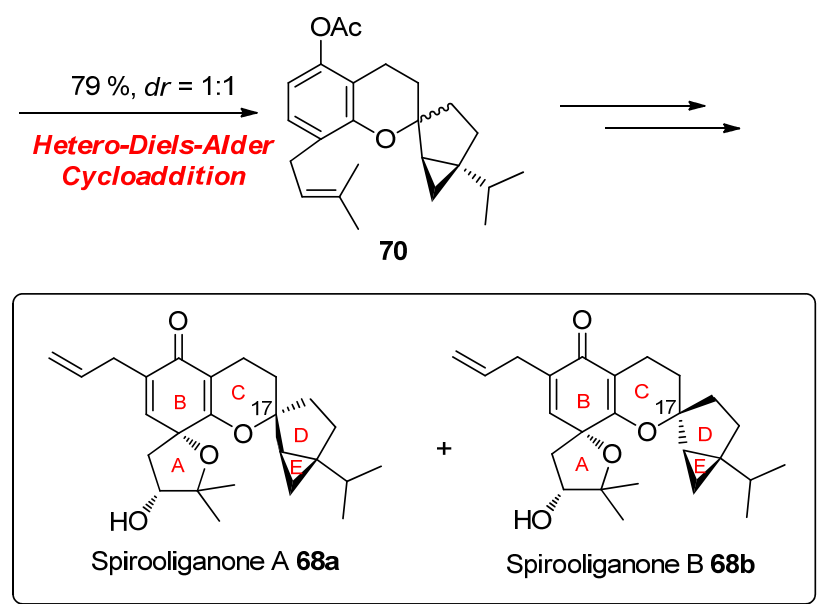

图式 18 天然产物 Spirooliganones A 和 B 的合成

Scheme 18 Synthesis of spirooliganones A and B

据其前体来决定，这在前面所介绍的合成实例中也有所 体现. 在反应中, 邻甲亚基环已二烯酮主要通过三种路 径来快速地再芳构化: 一是通过 Hetero-Diels-Alder reaction 过程, 这在很多含有螺环结构的天然产物中都 有应用，例如前面介绍到的 Berkelic acid、 $( \pm)$ Schefflone、Tocopherol trimers、Heliespirones A 和 C、 Variecolortides $\mathrm{A}$ 和 B 还有 Spirooliganones $\mathrm{A}$ 和 B 等实 例中均有体现; 二是通过 $6 \pi$-电环化的方法来再芳构化, 这在含有苯并吡喃结构的天然产物中多有应用, 例如前 面提到的 Huperguinone B 和 Tocopherol 都是经过这一途 径得到的; 第三是经过 Michael addition 过程再芳构化, 这个途径目前在天然产物合成中应用较少，就不多加举 例说明. 在这篇综述中我们主要介绍了邻甲亚基环已二 烯酮中间体在合成领域中的应用，在其他领域中因其独 特的性质以及高活泼性，其应用也在不断地被开发，例 如在化学生物学领域中, 已经被开发为一种高效的生物 正交反应 ${ }^{[45]}$, 对很多生物问题的探索提供了一种有效 的方法, 解释了一些生物学问题. 
总之，在有机合成中，邻甲亚基环已二烯酮作为一 个新发现的活泼中间体，目前在合成领域已做出了很多 贡献, 在以后的研究中, 关于它在这一领域的应用还会 有更大的开发潜力. 同时, 我们相信它也会在其他领域 发挥更多的作用，解释更多的科学问题.

\section{References}

[1] Fries, K. Liebigs Ann. Chem 1907, 339, 350.

[2] Chapman, O. L.; McIntosh, C. L. J. Chem. Soc. D 1971, 383.

[3] (a) Amouri, H.; Le Bras, J. Acc. Chem. Res. 2002, 35, 501.

(b) Amouri, H.; Besace, Y.; Bras, J. L.; Vaissermann, J. J. Am. Chem. Soc. 1998, 120, 6171.

[4] (a) Doria, F.; Richter, S. N.; Nadai, M.; Colloredo-Mels, S.; Mella, M.; Palumbo, M.; Freccero, M. J. Med. Chem. 2007, 50, 6570.

(b) Di Antonio, M.; Doria, F.; Richter, S. N.; Bertipaglia, C.; Mella, M.; Sissi, C.; Palumbo, M.; Freccero, M. J. Am. Chem. Soc. 2009, 131, 13132 .

(c) Van De Water, R. W.; Pettus, T. R. R. Tetrahedron 2002, 58, 5367.

(d) Pathak, T. P.; Sigman, M. S. J. Org. Chem. 2011, 76, 9210.

(e) Weinert, E. E.; Dondi, R.; Colloredo-Melz, S.; Frankenfield, K. N.; Mitchell, C. H.; Freccero, M.; Rokita, S. E. J. Am. Chem. Soc. 2006, 128, 11940.

(f) Wang, H.; Rokita, S. E. Angew. Chem., Int. Ed. 2010, 49, 5957.

[5] (a) Rodriguez, R.; Adlington, R. M.; Moses, J. E.; Cowley, A.; Baldwin, J. E. Org. Lett. 2004, 6, 3617.

(b) Gharpure, S. J.; Sathiyanarayanan, A. M.; Jonnalagadda, P. Tetrahedron Lett. 2008, 49, 2974.

(c) Rodriguez, R.; Moses, J. E.; Adlington, R. M.; Baldwin, J. E. Org. Biomol. Chem. 2005, 3, 3488.

[6] Chen, Y.; Steinmetz, M. G. J. Org. Chem. 2006, 71, 6053.

[7] (a) Yoshida, H.; Watanabe, M.; Fukushima, H.; Ohshita, J.; Kunai, A. Org. Lett. 2004, 6, 4049.

(b) McCrane, M. P.; Weinert, E. E.; Lin, Y.; Mazzola, E. P.; Lam, Y.-F.; Scholl, P. F.; Rokita, S. E. Org. Lett. 2011, 13, 1186.

(c) Tummatorn, J.; Ruchirawat, S.; Ploypradith, P. Chem.-Eur. J. 2010, $16,1445$.

(d) Samarakoon, T. B.; Hur, M. Y.; Kurtz, R. D.; Hanson, P. R. Org. Lett. 2010, 12, 2182.

[8] (a) Jones, R. M.; Selenski, C.; Pettus, T. R. R. J. Org. Chem. 2002, 67, 6911 .

(b) Kiselyov, A. S. Tetrahedron 2006, 62, 543.

(c) Lindsey, C. C.; Pettus, T. R. R. Tetrahedron Lett. 2006, 47, 201.

(d) Bray, C. D. Org. Biomol. Chem. 2008, 6, 2815.

[9] Sullivan, W. W.; Ullman, D.; Shechter, H. Tetrahedron Lett. 1969, $10,457$.

[10] (a) Liebner, F.; Schmid, P.; Adelwöhrer, C.; Rosenau, T. Tetrahedron 2007, 63, 11817.

(b) Bishop, L. M.; Winkler, M.; Houk, K. N.; Bergman, R. G.; Trauner, D. Chem.-Eur. J. 2008, 14, 5405.

(c) Patel, A.; Netscher, T.; Rosenau, T. Tetrahedron Lett. 2008, 49, 2442.

(d) Böhmdorfer, S.; Patel, A.; Netscher, T.; Gille, L.; Rosenau, T. Tetrahedron 2011, 67, 4858.

[11] (a) El Kaim, L.; Grimaud, L.; Oble, J. Org. Biomol. Chem. 2006, 4, 3410.

(b) Jiménez-Alonso, S.; Estévez-Braun, A.; Ravelo, Á. G.; Zárate, R.; López, M. Tetrahedron 2007, 63, 3066.

(c) Nandi, G. C.; Samai, S.; Kumar, R.; Singh, M. S. Tetrahedron
2009, 65, 7129 .

[12] (a) Ferreira, S. B.; da Silva, F. d. C.; Pinto, A. C.; Gonzaga, D. T. G.; Ferreira, V. F. J. Heterocycl. Chem. 2009, 46, 1080. (b) Willis, N. J.; Bray, C. D. Chem.-Eur. J. 2012, 18, 9160.

[13] Chapman, O.; Engel, M.; Springer, J.; Clardy, J. J. Am. Chem. Soc. 1971, 93, 6696.

[14] (a) Dai, M.; Wang, Z.; Danishefsky, S. J. Tetrahedron Lett. 2008, 49, 6613.

(b) Dai, M.; Danishefsky, S. J. Tetrahedron Lett. 2008, 49, 6610.

(c) Wang, Z.; Dai, M. J.; Park, P. K.; Danishefsky, S. J. Tetrahedron 2011, 67, 10249 .

[15] Rodriguez, R.; Adlington, R. M.; Moses, J. E.; Cowley, A.; Baldwin, J. E. Org. Lett. 2004, 6, 3617.

[16] (a) Snider, B. B.; Lu, Q. J. Org. Chem. 1994, 59, 8065. (b) Snider, B. B.; Lu, Q. J. Org. Chem. 1996, 61, 2839.

[17] Sierle, A. A.; Sierle, D. B.; Kelly, K. J. Org. Chem. 2006, 71, 5357.

[18] (a) Buchgraber, P.; Snaddon, T. N.; Wirtz, C.; Mynott, R.; Goddard, R.; Fürstner, A. Angew. Chem., Int. Ed. 2008, 47, 8450.

(b) Huang and Pettus reached a similar conclusion on the basis of a model study; see: Huang, Y.; Pettus, T. R. R. Synlett 2008, 1353.

(c) Wu, X.; Zhou, J.; Snider, B. B. Angew. Chem., Int. Ed. 2009, 48, 1283.

[19] Bender, C. F.; Yoshimoto, F. K.; Paradise, C. L.; Brabander, J. K. D. J. Am. Chem. Soc. 2009, 31, 11350.

[20] Yang, X.-L.; Hsieh, K.-L.; Liu, J.-K. Org. Lett. 2007, 9, 5135.

[21] Lawrence, A. L.; Adlington, R. M.; Baldwin, J. E.; Lee, V.; Kershaw, J. A.; Thompson, A. L. Org. Lett. 2010, 12, 1676.

[22] Bharate, S. B.; Singh, I. P. Tetrahedron Lett. 2006, 47, 7021.

[23] Nkunya, M. H. H.; Jonker, S. A.; de Gelder, R.; Wachira, S. W.; Kihampaa, C. Phytochemistry 2004, 65, 399.

[24] Row, L.-C.; Ho, J. C.; Chen, C. M. J. Nat. Prod. 2007, 70, 1214.

[25] Liao, D.; Li, H.; Lei, X. Org. Lett. 2011, 14, 18.

[26] Majumdar, N.; Korthals, K. A.; Wulff, W. D. J. Am. Chem. Soc. 2011, 134, 1357.

[27] George, J. H.; Hesse, M. D.; Baldwin, J. E.; Adlington, R. M. Org. Lett. 2010, 12, 3532.

[28] (a) Winkelmann, K.; Heilmann, J.; Zerbe, O.; Rali, T.; Sticher, O. J. Nat. Prod. 2000, 63, 104.

(b) Winkelmann, K.; Heilmann, J.; Zerbe, O.; Rali, T.; Sticher, O. J. Nat. Prod. 2001, 64, 701 .

(c) Winkelmann, K.; Heilmann, J.; Zerbe, O.; Rali, T.; Sticher, O. Helv. Chim. Acta 2001, 84, 3380.

[29] Crombie, L.; Jones, R. C. F.; Palmer, C. J. J. Chem. Soc., Perkin Trans. 1 1987, 317.

[30] Xiao, L.; Tan, W.; Li, Y. Synth. Commun. 1998, 28, 2861.

[31] (a) Macías, F. A.; Varela, R. M.; Torres, A.; Molinillo, J. M. G. Tetrahedron Lett. 1998, 39, 427.

(b) Macías, F. A.; Galindo, J. L. G.; Varela, R. M.; Torres, A.; Molinillo, J. M. G.; Fronczek, F. R. Org. Lett. 2006, 8, 4513.

[32] (a) Huang, C.; Liu, B. Chem. Commun. 2010, 46, 5280.

(b) Huang, C.; Zhang, W.; Liu, B. Sci. China: Chem. 2011, 54, 43.

(c) Sabui, S. K.; Venkateswaran, R. V. Tetrahedron 2003, 59, 8375.

(d) Doi, F.; Ogamino, T.; Sugai, T.; Nishiyama, S. Tetrahedron Lett. 2003, 44, 4877.

(e) Vyvyan, J. R.; Oaksmith, J. M.; Parks, B. W.; Peterson, E. M. Tetrahedron Lett. 2005, 46, 2457.

[33] Bai, W.-J.; Green, J. C.; Pettus, T. R. J. Org. Chem. 2011, 77, 379.

[34] Marsini, M. A.; Huang, Y.; Lindsey, C. C.; Wu, K.; Pettus, T. R. R. Org. Lett. 2008, 10, 1477.

[35] Kuttruff, C. A.; Zipse, H.; Trauner, D. Angew. Chem., Int. Ed. 2011, 50, 1402.

[36] Wang, W.-L.; Zhu, T.-J.; Tao, H.-W.; Lu, Z.-Y.; Fang, Y.-C.; Gu, 
Q.-Q.; Zhu, W.-M. Chem. Biodiversity 2007, 4, 2913.

[37] Laatsch, H.; Anke, H. Liebigs Ann. Chem. 1982, 2189.

[38] Nagasawa, H.; Isogai, A.; Suzuki, A.; Tamura, S. Tetrahedron Lett. 1976, 17, 1601 .

[39] Green, J. C.; Jiménez-Alonso, S.; Brown, E. R.; Pettus, T. R. Org. Lett. 2011, 13, 5500

[40] Harrison, B.; Crews, P. J. Org. Chem. 1997, 62, 2646.

[41] (a) Bhandari, P.; Crombie, L.; Harper, M. F.; Rossiter, J. T.; Sanders, M. D.; Whiting, A. J. Chem. Soc., Perkin Trans. 1 1992, 1685.

(b) Reisch, J.; Adebajo, A. C.; Kumar, V.; Aladesanmi, A. J. Phyto- chemistry 1994, 36, 1073.

[42] Luan, Y.; Sun, H.; Schaus, S. E. Org. Lett. 2011, 13, 6480.

[43] Ma, S.-G.; Gao, R.-M.; Li, Y.-H.; Jiang, J.-D.; Gong, N.-B.; Li, L.; Lü, Y.; Tang, W.-Z.; Liu, Y.-B.; Qu, J.; Lü, H.-N.; Li, Y.; Yu, S.-S. Org. Lett. 2013, 15, 4450.

[44] Song, L.; Yao, H.; Tong, R. Org. Lett. 2014, 16, 3740.

[45] (a) Li, Q.; Dong, T.; Liu, X.; Lei, X. J. Am. Chem. Soc. 2013, 135, 4996.

(b) Li, C.; Dong, T.; Li, Q.; Lei, X. Angew. Chem., Int. Ed. 2014, 53,12111 .

(Zhao, X.) 